\title{
Signal Design and Coding for High-Bandwidth OFDM in Car-to-Car Communications
}

\author{
Martin Braun*, Yves Koch*, Christian Sturm ${ }^{\ddagger}$, Friedrich K. Jondral* \\ ${ }^{*}$ Communications Engineering Lab, Karlsruhe Institute of Technology (KIT), Germany \\ ${ }^{\ddagger}$ Institut für Hochfrequenztechnik und Elektronik, Karlsruhe Institute of Technology (KIT), Germany \\ martin.braun@kit.edu, christian.sturm@kit.edu
}

\begin{abstract}
We analyse mobile propagation channels which occur in the context of car-to-car communications in the $24 \mathbf{~ G H z}$ ISM band. From the information gathered, we design robust frame designs for combined radar and communication systems using high-bandwidth OFDM signals. Two solutions are proposed: the combination of a spread spectrum technique and a standard convolutional encoder, as well as a low rate Reed-Muller code. We can show that while both perform well enough for the channels analysed, the Reed-Muller code has some advantages for the RF system, but achieves lower data rates.
\end{abstract}

\section{INTRODUCTION}

In the domain of traffic safety and intelligent transportation systems, two technologies are playing a lead role: radar systems, which provide additional sensory information about the surroundings including the other traffic, and car-to-car communication (C2C) systems, by which means vehicles can exchange information about traffic events, road safety information or manoeuvre intent. In a current research project the authors are analysing the means to merge these technologies. The ultimate goal of this project is to perform radar measurements and inter-vehicle communications with a single hardware and signal design, thus sharing hardware and frequencies between the two systems.

The proposed system uses OFDM signals for radar and communications and operates in the $24 \mathrm{GHz}$ ISM band. Sturm et al. describe the basics of the radar measurement in [1]. We present a detailed analysis of the physical OFDM parameter choice in [2]. In [3], we analyse the radar system from an estimation theoretic point of view and give some answers to the signal-to-noise ratio (SNR) performance of the radar, further restraining the OFDM parameter choice.

The choice of the signal bandwidth is a very important aspect in the system design. For the radar to work satisfactorily, the bandwidth must exceed $90 \mathrm{MHz}$ [2]. This imposes problems on the communication system: due to power restrictions ${ }^{1}$, the receiver must frequently operate with a low power density. Besides, the high bandwidth is not necessary for the communication system itself. As an example, assume one vehicle wishes to relay the information that it is about to perform an emergency brake. The information content is not very high, but it is vital that it gets transferred quickly and reliably. In particular, retransmissions are not desired since they can severely increase the transmission delay. The question

\footnotetext{
${ }^{1}$ E.g., in the EU, EIRP in the mentioned band is limited to $100 \mathrm{~mW}$.
}

arises how a signal with given bandwidth requirements can be designed such that reliability is not affected negatively.

In the proposed system, data is exchanged in form of short frames. Apart from the physical modulation parameters such as sub-carrier distance, which are discussed in [2], this paper will present the results of a short survey of suitable coding schemes. As an arbitrary limitation, the maximum communication distance for which the high reliability is designed for shall be fixed to $100 \mathrm{~m}$.

In the following Section, a brief description of the physical OFDM signals and an analysis of their performance in mobile channels is given. This will justify the need for robust signal and frame designs, as given in Section III. Section IV gives simulation results for the proposed design and a comparison to raw transmissions. Section V concludes.

\section{Mobile CHANNELS AND OFDM FADING}

\section{A. Communication System Description}

The analysed communication system uses a high-bandwidth OFDM signal with the parameters denoted in Table I. Every OFDM frame consists of $N \times M$ modulation symbols. The goal of the analysis is to first establish which values of SNR can be expected on each of the symbols, and then adapt the frame structure such that reliable data transmission is possible.

The identified solution must be able to work if only every $U$-th sub-carrier is used and the rest are set to zero, in an OFDMA fashion, since this is a possible way this system will be applied.

Synchronisation and equalisation issues will not be subject of analysis in this work. We will perform a best-case analysis, assuming that the synchronisation perfectly detects the timing and optimally aligns the centre frequency. In a similar fashion, equalisation at the receiver is assumed to be able to perfectly retrieve the phase. This way, the fading effects can be analysed separately from the issues of imperfect synchronisation or equalisation.

\section{B. Acquisition of channel data}

The wave propagation channels in the domain of $\mathrm{C} 2 \mathrm{C}$ systems are highly complex and are subject to heavy fading. The high number of reflecting objects, in particular other vehicles, leads to multipath propagation, resulting in frequency-selective fading, especially within urban areas. The potentially high velocities of the other participants as well as the continuous 


\begin{tabular}{r|l}
\hline Parameter & Value \\
\hline \hline Number of carriers $N$ & 1024 \\
\hline Number of symbols $M$ & 256 \\
\hline OFDM symbol duration $T$ & $11 \mu \mathrm{s}$ \\
\hline Guard interval fraction $G$ & $1 / 8$ \\
\hline Total OFDM Symbol duration $T_{O}$ & $(1+G) T=$ \\
& $12.375 \mu \mathrm{s}$ \\
\hline Total transmit power $P$ & $100 \mathrm{~mW}$ \\
\hline Noise Figure $\mathrm{NF}_{\mathrm{dB}}$ & $10 \mathrm{~dB}$ \\
\hline Center frequency $f_{c}$ & $24 \mathrm{GHz}$ \\
\hline Bumber of simultaneous accesses $U$ & 8 \\
\hline
\end{tabular}

Table I

OFDM SYSTEM PARAMETERS

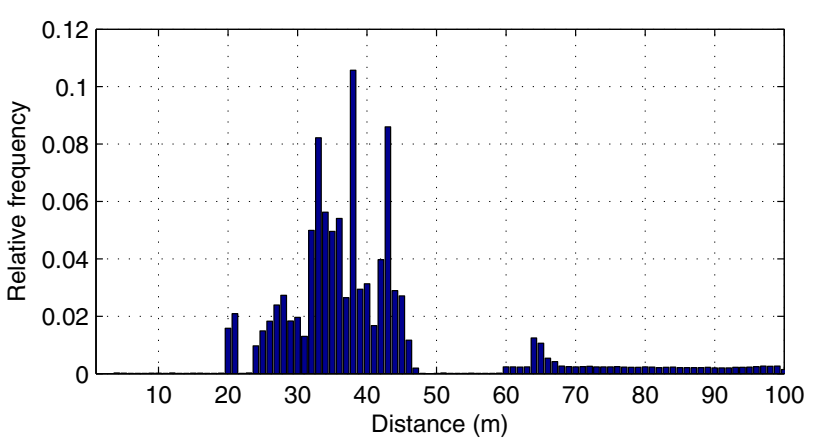

Figure 1. Histogram of vehicle distances in all available snapshots changing of the propagation environment lead to a timevariance of the channels.

Before the signals can be adapted to channels, some information about the channels needs to be acquired. For this work, we used a RayTracer to create channel data from simulated traffic. This method consists of two steps: first, a traffic scenario is generated, including roadside buildings, vegetation and moving vehicles, among which are the transmitting and receiving vehicle. Every vehicle is assigned a velocity. The position of every vehicle is re-calculated every $10 \mathrm{~ms}$ of simulation time, yielding a snapshot. In the second step, the wave propagation between two vehicles is calculated using ray tracing methods [4]. This process returns a list of $K$ propagation paths per snapshot, each with Doppler shift, time delay, attenuation and phase rotation. The received signal $r(t)$ can be calculated from this list according to

$$
r(t)=\sum_{k=0}^{K-1} \underline{a}_{k} s\left(t-\tau_{k}\right) e^{j 2 \pi f_{\mathrm{D}, k}\left(t-\tau_{k}\right)}
$$

where $\underline{a}_{k}$ is a complex attenuation factor, $\tau_{k}$ and $f_{\mathrm{D}, k}$ are the delay and Doppler shift of the $k$-th path, respectively, and $s(t)$ is the transmitted signal.

A total of 10 different traffic simulations was analysed, chosen such that a wide variety of traffic situations was included. Among these were two highway scenarios, where vehicles move at high velocities and eight urban scenarios with a variety of traffic and building densities. Any snapshots in which the distance between receiver and transmitter exceeded $100 \mathrm{~m}$ were discarded. A total of 10567 snapshots was finally used for analysis, yielding the same number of different channels.

It must be noted that the distances between the vehicles are not uniformly distributed; Figure 1 depicts their distribution. Distances ranging between 20 and $50 \mathrm{~m}$ occur most of the time, while shorter distances are underrepresented. However, data transmission between physically close vehicles is usually not very challenging to accomplish, and so this data set allows to focus on ranges with stronger fading.

\section{Doppler spread influence}

The first step in calculating SNR at the receiver is to analyse the influence of the Doppler spread. We will largely follow the work of Robertson and Kaiser [5] in this Subsection. A new variable, $f_{\text {sync }}$, is defined which denotes the frequency correction performed at the receiver. For any given channel, carrier-to-interference ratio due to Doppler spread and intercarrier interference (ICI) is [5]

$$
\gamma\left(f_{\text {sync }}\right)=\frac{\sum_{k=0}^{K-1}\left|\underline{a}_{k}\right|^{2} \operatorname{sinc}^{2}\left(\pi\left(f_{\mathrm{D}, k}-f_{\text {sync }}\right) T\right)}{\sum_{k=0}^{K-1}\left|\underline{a}_{k}\right|^{2}\left(1-\operatorname{sinc}^{2}\left(\pi\left(f_{\mathrm{D}, k}-f_{\mathrm{sync}}\right) T\right)\right)},
$$

where $\operatorname{sinc}(x)=\frac{\sin (x)}{x}$. Since we are performing a best-case analysis with respect to synchronisation, the final value for any given channel is

$$
\gamma_{\mathrm{opt}}=\max _{f_{\mathrm{sync}}} \gamma\left(f_{\mathrm{sync}}\right)
$$

The maximisation is performed numerically by use of an algorithm based on golden section search and parabolic interpolation. As the Doppler spread characteristics do not change within one channel in the applied model, there is only one scalar value $\gamma_{\text {opt }}$ per channel.

\section{Frequency selective OFDM frame fading}

Unlike the Doppler spread influence, the frequency selective fading must be calculated for every OFDM symbol and subcarrier. The OFDM signal was designed in a manner such that the channel coherence time is larger than one OFDM symbol [2]. As a result, the channel may be assumed time-invariant for the duration of one OFDM symbol and a time-invariant channel impulse response (CIR) is calculated for every OFDM symbol $m$ by fixing the Doppler shifts:

$$
h(t, m)=\sum_{k=0}^{K-1} \delta\left(t-\tau_{k}\right) \underline{a}_{k} e^{j 2 \pi\left(f_{\mathrm{D}, k}-f_{\mathrm{sync}}\right) m T_{O}} .
$$

The CIR is then sampled at an interval of $T_{s}=T / N$, corresponding to the sampling rate of the OFDM system. The time-discrete CIR for symbol $m$ is denoted as

$$
\mathbf{h}_{l, m}=h\left(l T_{s}, m\right)
$$

and further processed by a discrete Fourier transform (DFT) with respect to $l$, returning the complex attenuation of the $m$-th OFDM symbol and the $n$-th sub-carrier

$$
\mathbf{H}_{m, n}=\operatorname{DFT}\left\{\mathbf{h}_{l, m}\right\} .
$$




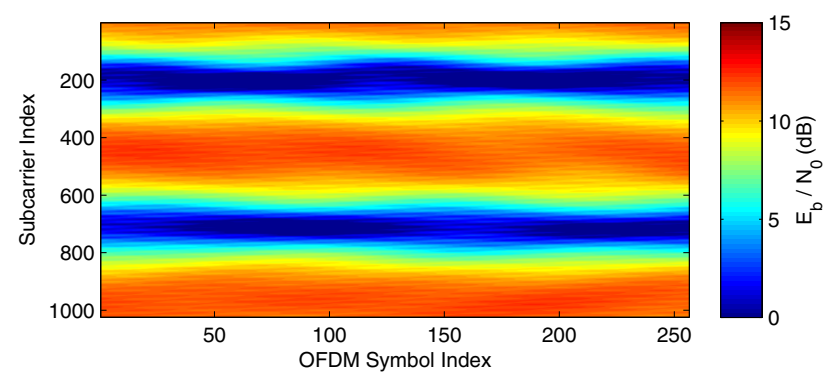

Figure 2. $E_{b} / N_{0}$ distribution over one OFDM frame

The bit energy on the $m$-th OFDM symbol and the $n$-th subcarrier is thus

$$
\left(\mathbf{E}_{b}\right)_{m, n}=E_{b, \mathrm{Tx}}\left|\mathbf{H}_{m, n}\right|^{2},
$$

where the bit energy at the transmitter is

$$
E_{b, T x}=\frac{P \cdot T \cdot U}{N} .
$$

\section{E. Total SNR}

The results from the previous sections enable us to calculate the SNR on every sub-carrier and symbol of an OFDM frame transmitted over a given channel. For brevity, SNR in $\mathrm{dB}$ is denoted by the matrix $\mathbf{S} \in \mathbb{R}^{N \times M}$,

$$
(\mathbf{S})_{n, m}=10 \log _{10}\left(\frac{\left(\mathbf{E}_{b}\right)_{n, m}}{\left(\mathbf{E}_{\mathrm{ICI}}\right)_{n, m}+N_{0}}\right) .
$$

SNR consists of the received bit energy and the sum of thermal noise and ICI energy. The thermal noise power density is calculated by $N_{0}=k_{B} \vartheta \cdot \mathrm{NF}$, where $\vartheta=290 \mathrm{~K}, k_{B}$ is the Boltzmann constant and NF is the linear noise figure of the receiver. The ICI energy is derived from (2), (3) and (7), resulting in

$$
\left(\mathbf{E}_{\mathrm{ICI}}\right)_{n, m}=\frac{\left(\mathbf{E}_{b}\right)_{n, m}}{\gamma_{\mathrm{opt}}}
$$

\section{F. Analysis}

Figure 2 shows the received SNR for one exemplary OFDM frame. This specific example illustrates the problems that need to be tackled. One can tell SNR varies over a range of several $\mathrm{dB}$ for different sub-carriers, from values close to $0 \mathrm{~dB}$, where BER is too high for reliable transmission, up to values greater than $10 \mathrm{~dB}$, where BER is small enough for a reliable communication link. Furthermore, the fading patterns change over time. It is not known a-priori which sub-carriers and which OFDM symbols are reliable, nor can the channel be measured before transmitting due to its rapid variation. The sub-carrier fading does also not necessarily have to vary as much as in the example shown, but can remain fairly constant at a high or low level.

To gather some statistics, $\mathbf{S}$ was calculated for every generated channel. Figure 3 shows the histogram of all entries of every $\mathbf{S}$ out of the 10567 channels.

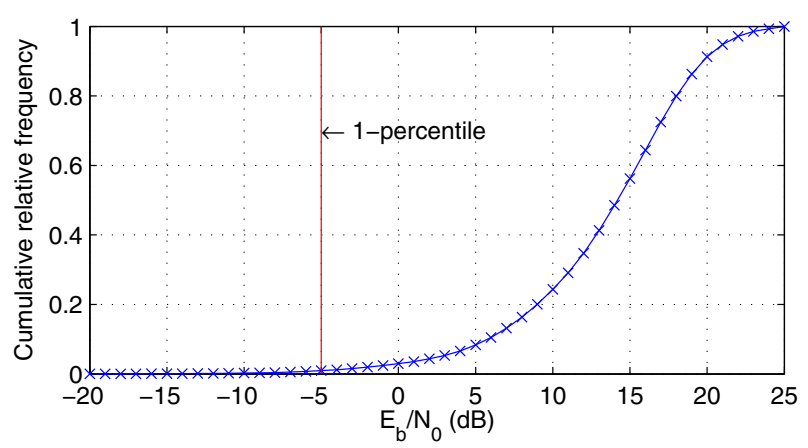

Figure 3. Cumulative distribution of $E_{b} / N_{0}$ values

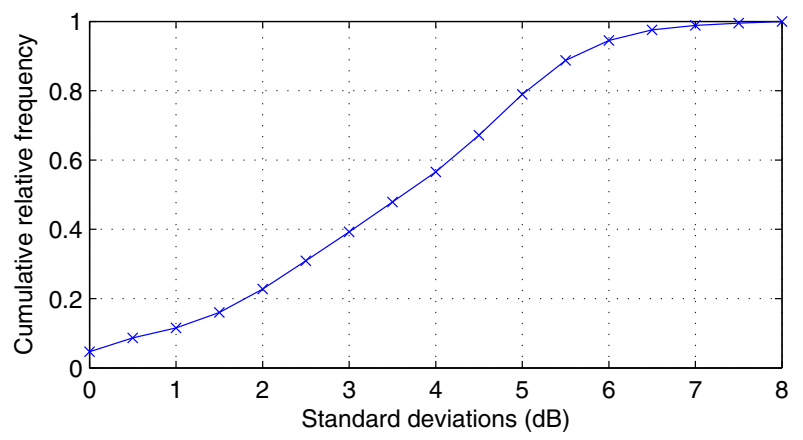

Figure 4. Cumulative distribution of $E_{b} / N_{0}$ standard deviations in one frame

In order to understand how the fading fluctuates within one frame, the empirical standard deviation

$$
\hat{\sigma}=\sqrt{\frac{1}{M N} \sum_{m=0}^{M-1} \sum_{n=0}^{N-1}\left((\mathbf{S})_{m, n}-\hat{\mu}\right)^{2}},
$$

where $\hat{\mu}$ is the arithmetic mean of all elements of $\mathbf{S}$, was calculated for every snapshot. A cumulative distribution of all standard deviations is shown in Figure 4. Within our set of channels, a standard deviation of $8 \mathrm{~dB}$ was never exceeded.

\section{Signal DeSign Proposal}

\section{A. Design goals}

Given the signal parameters and channel effects discussed in the previous Section, a signal design is to be created. The following guidelines are to be considered in the design process:

1) The maximum considered distance is $100 \mathrm{~m}$. This was already included in the channel analysis, see Section II.

2) Within range, the frame error rate shall not rise above $1 \%$. We assume a frame error as soon as one single bit of the received frame after decoding is wrong.

3) The design must be tolerant towards a wide range of signal-to-noise ratios and fading patterns. Additionally, it must be able to operate without any prior channel sounding. 


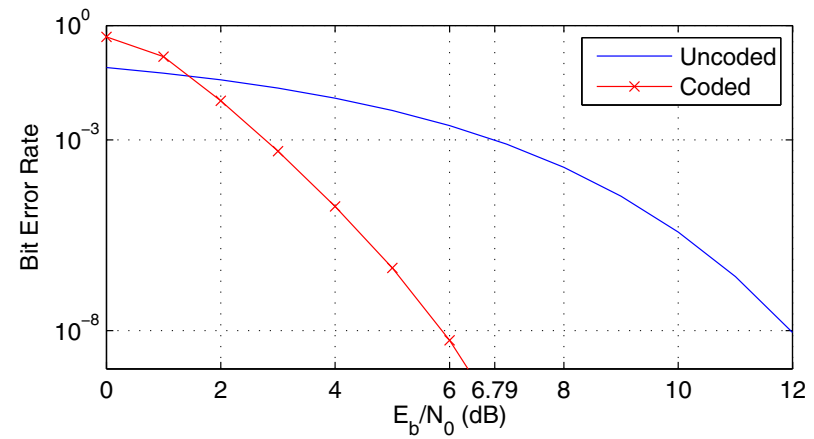

Figure 5. Performance of the convolutional code

In order to achieve the $1 \%$ frame error rate it is useful to find the 1-percentile in the cumulative distribution of the SNR values shown in Figure 3. A closer inspection of the empirical data reveals this to be at $-5 \mathrm{~dB}$, i.e. $99 \%$ of the received symbols have an SNR value of more than $-5 \mathrm{~dB}$.

In the next sections, two methods to achieve these goals are presented.

\section{B. Frequency domain spreading}

The first presented approach is to combine frequency domain spreading with a simple convolutional channel code. The information bits are first encoded using the industrystandard convolutional code of rate $R=1 / 2$ and the generator polynomials

$$
\begin{aligned}
& g_{1}(x)=1+x^{2}+x^{3}+x^{5}+x^{6} \\
& g_{2}(x)=1+x+x^{2}+x^{3}+x^{6}
\end{aligned}
$$

Figure 5 shows the performance of the given code when using soft-decision Viterbi decoding. Next, the code bits are modulated onto 16 sub-carriers each, spreading the symbols out in frequency domain. At the receiver, the de-spreading will ideally increase the available SNR by $10 \log _{10}(16)=12.04 \mathrm{~dB}$, while decreasing the maximum possible data rate. These values were chosen according to the following conservative estimates: In order to achieve the desired frame error rate, we target a BER of $10^{-8}$ in $99 \%$ of the time. The decoder requires an SNR of $6 \mathrm{~dB}$ to achieve this BER (see Figure 5). Before de-spreading, SNR must thus be $6 \mathrm{~dB}-12.04 \mathrm{~dB}=-6.04 \mathrm{~dB}$, which is below the 1-percentile. The factor 16 is the smallest integer factor of $N$ which achieves this.

This approach features two main advantages: first, it is very simple and easy to implement. De-spreading can be performed by a simple equal gain combining method, and the featured convolutional code is widely used, e.g. in the IEEE 802.11a standard. Besides, multi user access is also easy to integrate: instead of spreading onto 16 carriers, a shorter spreading sequence of $32 / U$ can be used to spread the energy onto every $U$-th carrier. The total bit energy at the receiver after the despreading is not affected by this, and the convolutional coder can be completely ignorant of $U$.
The achievable data rate of this system is

$$
r=\frac{1}{2} \cdot \frac{1}{16} \cdot \frac{N}{T_{O}} \approx 2.6 \mathrm{MBit} / \mathrm{s},
$$

although in a real system, synchronisation and equalisation will require pilot symbols which would further lower the real available throughput.

\section{Reed-Muller Coding}

An alternative approach is to solely rely on one type of channel coding with a very low code rate. The solution proposed here is the use of Reed-Muller-Codes (RM-Codes). The principal difficulty with OFDM is the high peak-to-mean envelope power ratio (PMEPR) of the output signal, which can be as high as the number of carriers in the systems. In [6], Popovic shows that the PMEPR of any Golay-sequence is bounded by a value of two (or $3 \mathrm{~dB}$ ). In [7] the connection between Golay-sequences and the second order Reed-Mullercode, which provides efficient encoding and decoding methods, was recognized. Very recently, the application of these codes in an OFDM radar context was also explained in [8].

The $r$-th order binary RM-code $R M_{2}(r, m)$ comprises $2^{k}$ codewords of length $2^{m}$, where $k$ is given by

$$
k=\sum_{i=0}^{r}\left(\begin{array}{c}
m \\
i
\end{array}\right) .
$$

Of particular importance is the code $R M_{2}(2, m)$ comprising $2^{\frac{m(m-1)}{2}}$ cosets of the code $R M_{2}(1, m)$, each containing $2^{m+1}$ codewords. By partitioning the codewords of $R M_{2}(2, m)$ into cosets of $R M_{2}(1, m)$, the codewords with large values of PMEPR can be isolated. If the transmitted codewords are restricted to those belonging to the Golay-cosets, the PMEPR is bounded by $3 \mathrm{~dB}$. For implementation convenience only one of the $m ! / 2$ Golay-cosets is used, which results in a code rate of $R_{c}=\frac{m+1}{2^{m}}$. As the length of the codewords increases, the code rate becomes very low.

If only one Golay-coset is used, the minimum Hamming distance of the resulting code is given by $d_{\min }=2^{m-1}$, whereas the minimum distance is $d_{\min }=2^{m-2}$ if two or more Golay-cosets are used. Another advantage of using only one Golay-coset is the reduction of the complexity of the decoder. After subtracting the Golay-coset representative of received codeword, the result can be decoded with a basic $R M_{2}(1, m)$ decoder. If a large number of cosets is used, this operation has to be done for every coset representative, which increases the complexity. The binary Reed-Muller-code of first order $R M_{2}(1, m)$ can be maximum-likelihood decoded using the fast Hadamard transform (FHT), which can be implemented in hardware [9]. Furthermore, the decoder can be realised as hard- or soft-decision decoder without extra complexity.

Since we only want to use every $U$-th sub-carrier, we can use the RM-code with $m=7$, resulting in one code word of length $n=128$ per OFDM symbol, thereby guaranteeing the PMEPR of $3 \mathrm{~dB}$. 


\begin{tabular}{r|c|c} 
& Frequency Domain Spreading & RM-Coding \\
\hline \hline Urban & 0 & 0 \\
\hline Highway & $1.32 \cdot 10^{-3}$ & $2.30 \cdot 10^{-3}$ \\
\hline Total & $1.24 \cdot 10^{-4}$ & $2.13 \cdot 10^{-4}$ \\
\hline \multicolumn{3}{c}{ Table II } \\
FRAME ERROR RATES
\end{tabular}

Every error of weight smaller than half the Hamming distance $w t(e)<2^{m-2}=32$ can be corrected if one Golaycoset is used. If the BER before the decoder is given by $p$, the frame error rate is thus

$$
P_{\text {frameerr }}=1-\left(F_{p, n}(32)\right)^{M}
$$

where $F_{p, n}(x)$ is the CDF of a binomial distribution with parameters $p$ and $n$. As an example, assume the SNR before the receiver is $-5 \mathrm{~dB}$. Since every $U$-th sub-carrier is used, this value is increased by $10 \log _{10}(8)=9 \mathrm{~dB}$ up to $4 \mathrm{~dB}$. At $4 \mathrm{~dB}$, the BER in AWGN is $p=0.0125$, yielding a near-zero value of $P_{\text {frameerr }}$.

The achievable data rate of the system using the parameters of Table I is

$$
r=\frac{N}{U} \cdot R_{c} \cdot \frac{1}{T_{O}} \approx 0.64 \mathrm{MBit} / \mathrm{s},
$$

although, as we mention before, in reality it will be smaller due to pilot symbols. It must be noted that this data rate is only $25 \%$ that of the previous system.

\section{Simulation Results}

We performed simulations to test the effectiveness of the methods in real channels. It must be noted that these simulations are, by themselves, not adequate to fully assess the quality of the coding methods, since it is unclear how much they degrade in adverse synchronisation or equalisation. However, these simulations provide some useful measures if the theoretical analysis of the chosen codes is sensible at all.

The simulations consisted of the following steps:

- For every channel, 16 OFDM frames constructed according to the parameters in Table I were created. The data loaded into the frame was created according to the coding methods described in the previous Section.

- Likewise, the matrix $\mathbf{S}$ was calculated.

- For every element of the OFDM frames, white Gaussian noise was added such that the total SNR was consistent with the values in $\mathbf{S}$.

- Every frame was demodulated and decoded according the given method.

Table II shows the results and confirms the applicability of the codes. The desired frame error rate was achieved by a wide margin. The advantages of each presented method are clear: The frequency domain spreading approach can achieve higher data rates, while the RM-coding approach has lower requirements towards the RF hardware.

\section{CONCLUSION}

In this paper, we present two possibilities to package OFDM frames for use in combined OFDM radar and communication in the $24 \mathrm{GHz}$ ISM band. The methods differ in several respects. The combined spreading and convolutional coding yields a higher data rate and the employed coding scheme is very common. However, the Reed-Muller code makes the RF front-end easier to implement since due to the constant low PMEPR the transmitter linearity requirements are lower. Its decoding scheme is based on the computationally efficient FHT. Both codes have suitable error correction capabilities.

Of course these two methods do not exhaust the possibilities, and many other codes could be applied. Our goal was to present two different available paths. Future development of the combined OFDM radar and communications system must clearly define priorities concerning data rate, RF frontend complexity and robustness, thereby making a choice for one of the methods.

In a next step, synchronisation and equalisation must be taken into account and full simulations will be performed. For a final and conclusive evaluation of the signal designs, it will be necessary to perform live measurements as well. In [10], we have presented a measurement setup which could be used to run live measurements.

\section{ACKNOWLEDGEMENTS}

The authors gratefully acknowledge that their work is supported within the priority program No. 1163 (TakeOFDM) by the German Research Foundation (DFG).

\section{REFERENCES}

[1] C. Sturm, E. Pancera, T. Zwick, and W. Wiesbeck, "A Novel Approach to OFDM Radar Processing," Radar Conference, IEEE, May 2009.

[2] M. Braun, C. Sturm, A. Niethammer, and F. K. Jondral, "Parametrization of Joint OFDM-based Radar and Communication Systems for Vehicular Applications," 20th IEEE Symposium on Personal Indoor and Mobile Radio Communications, 2009.

[3] M. Braun, C. Sturm, and F. K. Jondral, "Maximum Likelihood Speed and Distance Estimation for OFDM Radar," Radar Conference, IEEE International, 2010.

[4] J. Maurer, "Strahlenoptisches Kanalmodell für die Fahrzeug-FahrzeugFunkkommunikation," Ph.D. dissertation, Karlsruhe, 2005, available online at www.ubka.uni-karlsruhe.de.

[5] P. Robertson and S. Kaiser, "Analysis of Doppler Spread Perturbations in OFDM(A) Systems," European Transactions on Telecommunications, vol. 11, pp. 585-592, Nov./Dec. 2000.

[6] B. Popovic, "Synthesis of power efficient multitone signals with flat amplitude spectrum," IEEE Transactions on Communications, vol. 39, no. 1 , July 1991.

[7] J. A. Davis and J. Jedwab, "Peak-to-mean power control in OFDM, Golay complementary sequences and Reed-Muller codes," IEEE Transactions on Information Theory, vol. 45, no. 7, November 1999.

[8] Y. Paichard, "OFDM Waveforms for Multistatic Radars," Radar Conference, IEEE International, May 2010.

[9] F. MacWilliams and N. Sloane, The Theory of Error-Correcting Codes. North-Holland Mathematical Library, 1977.

[10] C. Sturm, M. Braun, T. Zwick, and W. Wiesbeck, "Performance Verification of Symbol-Based OFDM Radar Processing," Radar Conference, IEEE International, 2010. 